\title{
Second order subexponential distributions with finite mean and their applications to subordinated distributions
}

\author{
Jianxi Lin* \\ Mathematics School of Xiamen University, Xiamen, Fujian 361005, China.
}

September 27, 2018

\begin{abstract}
Consider a probability distribution subordinate to a subexponential distribution with finite mean. In this paper, we discuss the second order tail behavior of the subordinated distribution within a rather general framework in which we do not require the existence of density functions. For this aim, the so-called second order subexponential distribution is proposed and some related properties of its are established. Our results unified and improved some classical results.
\end{abstract} Keywords: Second order tail behaviour; heavy-tailed distribution; subexponential distribution; subordinated distribution; convergence rate

\section{Introduction}

Let $N$ be a non-negative integer valued random variable with distribution $\left\{p_{n}\right\}_{n \geq 0}$ and $X_{1}$, $X_{2}, \cdots$ be a sequence of non-negative i.i.d. random variables, independent of $N$. The common distribution of $X_{i}$ 's is denoted by $F$. Define for $n \geq 1$,

$$
S_{n}:=\sum_{k=1}^{n} X_{k},
$$

and $S_{0}=0$. In many fields of applied probability, one has to investigate the tail behavior of $S_{N}$, whose distribution is equal to

$$
G(x):=\sum_{n=0}^{\infty} p_{n} F^{n *}(x),
$$

where $F^{* 0}$ is the unit mass at zero and for $n \geq 1, F^{* n}$ denotes the $n$-fold convolution of distribution $F$. Obviously, $G$ is a probability distribution subordinate to $F$ with subordinator $\left\{p_{n}\right\}_{n \geq 0}$.

Denote the tail of distribution $G$ by $\bar{G}(x)=G(x, \infty)=1-G(x)$. A first order approximation to $\bar{G}(x)$ as $x \rightarrow \infty$ has been considered by Chistyakov [7], in which he introduce the so-called

\footnotetext{
*Author's Email address: linjx999@gmail.com
} 
subexponential distribution class $\mathscr{S}$. By definition, a distribution $F$ on $[0, \infty)$ is said to belong to the class $\mathscr{S}$ if for $n=2$ (hence for all $n \geq 2$ ),

$$
\lim _{x \rightarrow \infty} \frac{\overline{F^{n *}}(x)}{\bar{F}(x)}=n
$$

Chistyakov [7] states that if $F \in \mathscr{S}$ and $E\left(z^{N}\right)$ is analytic at $z=1$, then

$$
\bar{G}(x) \sim\left(\sum_{n=0}^{\infty} n p_{n}\right) \bar{F}(x), \quad x \rightarrow \infty
$$

where, here and throughout the paper, we write $a(x) \sim b(x), x \rightarrow \infty$ to denote

$$
\lim _{x \rightarrow \infty} \frac{a(x)}{b(x)}=1 .
$$

Many papers have been devoted to investigating the convergence rate in (1.4); See Omey and Willekens [13][14], Omey [15], Baltrūnas and Omey [3][4]], Baltrūnas et al. [5], Geluk and Pakes [9], and Geluk [10][11], among others. In these papers, the precise convergence rate as well as the O-type results has been considered. Generally speaking, the results about the convergence rate in (1.4) would be different according to whether or not the distribution $F$ has a finite mean.

Denote the mean of $F$ by $\mu$. In this paper, we assume $\mu<\infty$ and focus on the precise convergence rate in (1.4). Most of the related results usually assume the existence of the density of $F$. For example, a result from Omey [14] requires $F$ to have a subexponential density $f$. By definition, the density $f$ is said to be a subexponential density, denoted by $f \in \mathcal{S} d$, if

$$
\lim _{x \rightarrow \infty} \frac{f(x+y)}{f(x)}=1, \quad \forall y \in \mathbb{R}
$$

and

$$
\lim _{x \rightarrow \infty} \frac{\int_{0}^{x} f(y) f(x-y) d y}{f(x)}=2 .
$$

The first part of Theorem 2.2(ii) in Omey and Willekens [14] is stated as follows.

Theorem 1.1.(Omey and Willekens [14]) Suppose $E\left(z^{N}\right)$ is analytic at $z=1, f \in \mathcal{S} d$, and

$$
\overline{F^{2 *}}(x)-2 \bar{F}(x) \sim 2 \mu f(x), \quad x \rightarrow \infty,
$$

then

$$
\bar{G}(x)-\left(\sum_{n=0}^{\infty} n p_{n}\right) \bar{F}(x) \sim\left\{\mu \sum_{n=0}^{\infty} n(n-1) p_{n}\right\} f(x), \quad x \rightarrow \infty
$$


Efforts have been taken by Omey [15] to remove the condition of densities in Theorem 1.1; See Theorem 6.1 of Omey [15]. However the condition imposed there requires $F$ to belong to a subclass of the distributions with both dominatedly varying tails and long tails (see Omey [15] for details).

In this paper, we aim to generalize Theorem 1.1 to the case where the density of $F$ does not necessarily exist. One main result of ours (see Theorem 2.1) unifies Theorem 1.1 and the related result in Omey [15]. The appropriate condition for our result is expressed in terms of some class of distributions, which we call the second order subexponential distribution class. Its definition and properties are also stated in section 2 as main results. The proofs are given in section 3 .

\section{Main results}

Let $t \in(0, \infty]$ and write $\Delta(t)=(0, t]$,

$$
x+\Delta(t)=(x, x+t]
$$

and

$$
F(x+\Delta(t))=F(x, x+t]=F(x+t)-F(x) .
$$

The so-called local subexponential class as well as the local long-tailed class is introduced by Asmussen et al. [1]. By definition, a distribution $F$ on $[0, \infty)$ is said to belong to the local long-tailed class $\mathscr{L}_{\Delta(t)}$, if the relation

$$
F(x+y+\Delta(t)) \sim F(x+\Delta(t)), \quad x \rightarrow \infty
$$

holds uniformly in $y \in[0,1]$ and hence, it holds uniformly on any finite interval of $y$. Furthermore, $F$ is said to belong to the local subexponential class $\mathscr{S}_{\Delta(t)}$, if $F \in \mathscr{L}_{\Delta(t)}$ and

$$
F^{* 2}(x+\Delta(t)) \sim 2 F(x+\Delta(t)), \quad x \rightarrow \infty .
$$

Definition 2.1. We say a distribution $F$ on $[0, \infty)$ with finite mean $\mu$ belongs to the second order subexponential class $\mathscr{S}_{2}$, iffor all $t \in(0, \infty), F \in \mathscr{S}_{\Delta(t)}$ and

$$
\overline{F^{2 *}}(x)-2 \bar{F}(x) \sim 2 \mu F(x, x+1], \quad x \rightarrow \infty .
$$

Proposition 2.1. (1) Assume $F \in \mathscr{S}_{2}$, then for all $n \geq 2$,

$$
\overline{F^{n *}}(x)-n \bar{F}(x) \sim n(n-1) \mu F(x, x+1], \quad x \rightarrow \infty .
$$

(2) Assume for $F \in \mathscr{L}_{\Delta(t)}$ all $t \in(0, \infty), \mu<\infty$ and $\bar{F}^{2}(x)=o(F(x, x+1])$ (it means that $\left.\lim _{x \rightarrow \infty} \bar{F}^{2}(x) / F(x, x+1]=0\right)$. If for some $n \geq 2$, the relation $(2.4)$ holds, then $F \in \mathscr{S}_{2}$. 
An uniform bound for (2.4) is given as follows.

Proposition 2.2. Assume $F \in \mathscr{S}_{2}$, then for every fixed $\varepsilon>0$, there exist constants $A, K>0$, which are independent of $n$, such that for all $n \geq 2$,

$$
\sup _{x \geq A}\left|\frac{\overline{F^{n *}}(x)-n \bar{F}(x)}{F(x, x+1]}\right| \leq K(1+\varepsilon)^{n} .
$$

Our next result investigates the second order tail behaviour of $G$.

Theorem 2.1. (1) If $F \in \mathscr{S}_{2}$ and $E\left(z^{N}\right)$ is analytic at $z=1$, then

$$
\bar{G}(x)-\left(\sum_{n=0}^{\infty} n p_{n}\right) \bar{F}(x) \sim\left\{\mu \sum_{n=0}^{\infty} n(n-1) p_{n}\right\} F(x, x+1], \quad x \rightarrow \infty .
$$

(2) Suppose $F \in \mathscr{L}_{\Delta(t)}$ for all $t \in(0, \infty), \mu<\infty$ and $\bar{F}^{2}(x)=o(F(x, x+1])$. If the relation (2.6) holds and there exists some $l \geq 2$ such that $p_{l}>0$, then $F \in \mathscr{S}_{2}$.

Remark 2.1. As has been shown by Asmussen et al. [1], if $F$ has a density $f \in \mathcal{S} d$, then for all $t \in(0, \infty), F \in \mathscr{S}_{\Delta(t)}$. Hence Theorem 2.1 improves Theorem 2.2(ii) of Omey and Willekens [14]. By Corollary 2.1 (see below), we know that Theorem 2.1 also improves Theorem 6.1 of Omey [15] in the case $\mu<\infty$.

Next we present a result on tail equivalences.

Proposition 2.3. Let $F$ and $H$ be two distributions. If $F \in \mathscr{S}_{2}$ and there exist constants $K>0$, $c \in \mathbb{R}$ such that

$$
\frac{\bar{H}(x)-K \bar{F}(x)}{F(x, x+1]} \rightarrow c, \quad x \rightarrow \infty
$$

then $H \in \mathscr{S}_{2}$.

Remark 2.2. From Proposition 2.3. we know that (2.7) defines a class of distribution that is equivalent to $F$. In this equivalent class, there must exist a distribution that satisfies (2.7) and has a subexponential density. To see this, let $K=1 / \int_{0}^{1} \bar{F}(s) d$ s and define

$$
\widetilde{h}(x):=K F(x, x+1], \quad \forall x>0 .
$$

Assume $F \in \mathscr{S}_{\Delta(t)}$ for all $t \in(0, \infty)$. In view of Lemma 2.1 below, we have $\widetilde{h} \in \mathcal{S} d$. Denote the distribution function of $\widetilde{h}(x)$ by $H$. It is easy to see

$$
\int_{x}^{\infty} F(s, s+1] d s=\int_{x}^{x+1} \bar{F}(s) d s=\int_{0}^{1} \bar{F}(x+z) d z .
$$


Since $F \in \mathscr{L}_{\Delta(t)}$ for all $t \in(0, \infty)$, then it follows from Lemma 3.1 below and the dominated convergence theorem that

$$
\begin{aligned}
\bar{H}(x)-K \bar{F}(x) & =-K \int_{0}^{1}(\bar{F}(x)-\bar{F}(x+z)) d z \\
& \sim-K F(x, x+1] \int_{0}^{1} z d z \\
& =-\frac{K}{2} F(x, x+1]
\end{aligned}
$$

i.e., $H$ satisfies (2.7) with $c=-K / 2$.

Remark 2.3. It follows from Proposition 2.1 1) that in Proposition 2.3 (2.7) implies that (2.7) holds with $H$ and $F$ (in the numerator) replaced by $H^{n *}$ and $F^{n *}$ and c replaced by $n c+K\left(\mu_{H}-\right.$ $\mu) n(n-1)$, where $\mu_{H}=\int_{0}^{\infty} \bar{H}(x) d x<\infty$.

The following lemma about local subexponential distributions, which is cited by Remark 2.2 might be of independent interest.

Lemma 2.1. Let $t \in(0, \infty)$ be fixed, then $F \in \mathscr{S}_{\Delta(t)}$ if and only if $K F(\cdot+\Delta(t)) \in \mathcal{S} d$, where $K$ as a positive constant, is defined as

$$
K=\frac{1}{\int_{0}^{t} \bar{F}(s) d s} .
$$

Finally, we give some sufficient conditions for $F \in \mathscr{S}_{2}$. A distribution $F$ on $[0, \infty)$ is said to belong to $\mathscr{S}^{*}$ (see Klüppelberg [12]), if

$$
\int_{0}^{x} \bar{F}(y) \bar{F}(x-y) d y \sim 2 \mu \bar{F}(x), \quad x \rightarrow \infty .
$$

It is well known that $\mathscr{S}^{*} \subset \mathscr{S}$. Denote $h(x)=F(x, x+1]$ and $q(x)=h(x) / \bar{F}(x)$.

Proposition 2.4. Suppose $F \in \mathscr{L}_{\Delta(t)}$ for all $t \in(0, \infty), \mu<\infty, F \in \mathscr{S}^{*}, \bar{F}^{2}(x / 2)=o(F(x, x+1])$ and for all $y>0$,

$$
\limsup _{x \rightarrow \infty} \frac{q(x y)}{q(x)}<\infty
$$

Then $F \in \mathscr{S}_{2}$.

Remark 2.4. In view of Proposition 2.1 1), we know that Proposition 2.4 improves Proposition 3.5(iii) of Baltrūnas [2]. 
Corollary 2.1. Suppose $F \in \mathscr{L}_{\Delta(t)}$ for all $t \in(0, \infty), \mu<\infty, \bar{F}^{2}(x / 2)=o(F(x, x+1])$ and for all $y>0$,

$$
\limsup _{x \rightarrow \infty} \frac{h(x y)}{h(x)}<\infty .
$$

Then $F \in \mathscr{S}_{2}$.

Some typical subexponential distributions including the Pareto, lognormal and Weibull (with parameter between 0 and 1) distributions all belong to $\mathscr{S}_{2}$, which is shown in the following.

For the Pareto distribution $F$, i.e., $\bar{F}(x)=c x^{-\alpha}$, where $c>0$ and $\alpha>1$, it is easy to obtain for every fixed $t \in(0, \infty)$,

$$
F(x, x+t] \sim \operatorname{cotx}^{-(\alpha+1)}, \quad x \rightarrow \infty
$$

and hence by Corollary 2.1, it is easy to see $F \in \mathscr{S}_{2}$.

Let $F$ be the lognormal distribution with the density $f(x)=e^{-(\ln x-\mu)^{2} / 2 \sigma^{2}} / x \sqrt{2 \pi \sigma^{2}}$. Let $\Phi$ be the standard normal distribution with the density $\phi$. Then by using the relation between the lognormal and normal distributions, and the following well-known relation

$$
1-\Phi(x) \sim \frac{1}{x} \phi(x), \quad x \rightarrow \infty
$$

it is easy to obtain

$$
\bar{F}(x)=1-\Phi\left(\frac{\ln x-\mu}{\sigma}\right) \sim \frac{\sigma}{\ln x} \phi\left(\frac{\ln x-\mu}{\sigma}\right), \quad x \rightarrow \infty .
$$

On the other hand, it is easy to see for every fixed $t \in(0, \infty)$,

$$
F(x, x+t] \sim t f(x)=\frac{t}{x \sigma} \phi\left(\frac{\ln x-\mu}{\sigma}\right), \quad x \rightarrow \infty .
$$

Thus,

$$
q(x) \sim \frac{\ln x}{x} \cdot \frac{1}{\sigma^{2}}, \quad x \rightarrow \infty .
$$

By Proposition 2.4, it is easy to see $F \in \mathscr{S}_{2}$.

For the Weibull distribution $F$, i.e., $\bar{F}(x)=e^{-x^{\beta}}, \beta \in(0,1)$, we have for every fixed $t \in(0, \infty)$,

$$
F(x, x+t]=\beta t x^{\beta-1} e^{-x^{\beta}}, \quad x \rightarrow \infty .
$$

Hence

$$
q(x) \sim \beta x^{\beta-1}, \quad x \rightarrow \infty .
$$

By Proposition 2.4, it is easy to see $F \in \mathscr{S}_{2}$.

A distribution, which belongs to $\mathscr{S}_{2}$ but does not have a density, is presented in the following example. 
Example 2.1. Define for $n \geq 2$,

$$
\bar{F}(x)=c\left(1+\frac{1}{n}\right) x^{-\alpha}, \quad n^{\beta} \leq x<(n+1)^{\beta},
$$

where $c>0, \alpha>1$ and $\beta \in(1,2)$. Since

$$
(n+1)^{\beta}-n^{\beta}=n^{\beta}\left[\left(1+\frac{1}{n}\right)^{\beta}-1\right] \sim \beta n^{\beta-1} \rightarrow \infty, \quad n \rightarrow \infty,
$$

then for any fixed $t \in(0, \infty)$ and sufficiently large $x$, there only exist two cases: $n^{\beta} \leq x<x+t<$ $(n+1)^{\beta}$ or $n^{\beta} \leq x<(n+1)^{\beta} \leq x+t<(n+2)^{\beta}$ for some $n$. In either case, through some simple calculations, it is to easy see that the relation (2.15) always holds. From this and in view of

$$
\bar{F}(x) \sim c x^{-\alpha}, \quad x \rightarrow \infty,
$$

it is easy to see that the conditions of Corollary 2.1 are satisfied, and thus, $F \in \mathscr{S}_{2}$. However, since $F$ is not continuous, it does not have a density.

\section{Proofs}

In the sequel, all limit relations between two functions $g_{1}(x)$ and $g_{2}(x)$ of one variable $x$, unless explicitly stated otherwise, are for $x \rightarrow \infty$. If $g_{1}$ or $g_{2}$ is a function of two variables $x$ and $A$, then the limit relations between them, unless explicitly stated otherwise, are for $x \rightarrow \infty$ and then $A \rightarrow \infty$, the meaning of which is specified as follows:

$g_{1}=o\left(g_{2}\right)$ denotes

$$
\lim _{A \rightarrow \infty} \limsup _{x \rightarrow \infty}\left|g_{1} / g_{2}\right|=0
$$

$g_{1} \sim g_{2}$ denotes

$$
\lim _{A \rightarrow \infty} \limsup _{x \rightarrow \infty}\left|g_{1} / g_{2}-1\right|=0
$$

$g_{1} \lesssim g_{2}$ denotes

$$
\underset{A \rightarrow \infty}{\limsup } \limsup _{x \rightarrow \infty} g_{1} / g_{2}<\infty \text {; }
$$

$g_{1} \gtrsim g_{2}$ denotes

$$
\liminf _{A \rightarrow \infty} \liminf _{x \rightarrow \infty} g_{1} / g_{2}>0 .
$$


Lemma 3.1. Assume $F \in \mathscr{L}_{\Delta(t)}$ for all $t \in(0, \infty)$. Then for all $t \in(0, \infty)$,

$$
\frac{F(x+\Delta(t))}{F(x, x+1]} \rightarrow t, \quad x \rightarrow \infty .
$$

Proof. For any $\delta \in(0, \min \{t, 1\})$, there exist positive integers $k, n$ such that

$$
k \delta \leq t<(k+1) \delta, \quad n \delta \leq 1<(n+1) \delta .
$$

Obviously, when $\delta \rightarrow 0+$,

$$
k \sim \frac{t}{\delta}, \quad n \sim \frac{1}{\delta}
$$

Obviously,

$$
\begin{aligned}
& \sum_{i=1}^{k} F(x+(i-1) \delta, x+i \delta] \leq F(x+\Delta(t)) \leq \sum_{i=1}^{k+1} F(x+(i-1) \delta, x+i \delta], \\
& \sum_{i=1}^{n} F(x+(i-1) \delta, x+i \delta] \leq F(x, x+1] \leq \sum_{i=1}^{n+1} F(x+(i-1) \delta, x+i \delta] .
\end{aligned}
$$

Let $\delta$ be fixed, then for all $i=1,2, \cdots, \max \{k, n\}$,

$$
F(x+(i-1) \delta, x+i \delta] \sim F(x, x+\delta],
$$

and hence

$$
\frac{k}{n+1} \leq \liminf _{x \rightarrow \infty} \frac{F(x+\Delta(t))}{F(x, x+1]} \leq \limsup _{x \rightarrow \infty} \frac{F(x+\Delta(t))}{F(x, x+1]} \leq \frac{k+1}{n} .
$$

Let $\delta \rightarrow 0+$ in (3.6) and in view of (3.3), we obtain (3.1).

Lemma 3.2. For any $t \in(0, \infty)$, the following three assertions are equivalent:

(1) $F \in \mathscr{S}_{\Delta(t)}$,

(2) $F \in \mathscr{L}_{\Delta(t)}$ and

$$
\int_{0}^{x-A} F(x-y+\Delta(t)) d F(y) \sim F(x+\Delta(t)),
$$

(3) $F \in \mathscr{L}_{\Delta(t)}$ and

$$
\int_{A}^{x-A} F(x-y+\Delta(t)) d F(y)=o(F(x+\Delta(t))) .
$$

Proof. The proof of this lemma is similar to that of Proposition 2 of Asmussen et al. [1], so we omit it. 
Lemma 3.3. Assume $F \in \mathscr{L}_{\Delta(t)}$ for all $t \in(0, \infty)$ and $\mu<\infty$. Then the relation (2.3) is equivalent to

$$
\int_{A}^{x-A}\{\bar{F}(x-y)-\bar{F}(x)\} d F(y)-\bar{F}^{2}(x)=o(F(x, x+1]) .
$$

Proof. Assume $F \in \mathscr{L}_{\Delta(t)}$ for all $t \in(0, \infty)$ and $\mu<\infty$. Notice that

$$
\overline{F^{2 *}}(x)-2 \bar{F}(x)=\int_{0}^{x}\{\bar{F}(x-y)-\bar{F}(x)\} d F(y)-\bar{F}^{2}(x) .
$$

By Lemma 3.1, it is obvious that

$$
\int_{0}^{A}\{\bar{F}(x-y)-\bar{F}(x)\} d F(y) \sim \int_{0}^{\infty} y d F(y) \cdot F(x, x+1]=\mu F(x, x+1] .
$$

By integrating by parts, we obtain

$$
\begin{aligned}
& \int_{x-A}^{x}\{\bar{F}(x-y)-\bar{F}(x)\} d F(y) \\
= & \int_{0}^{A}\{\bar{F}(x-y)-\bar{F}(x)\} d F(y)+\{\bar{F}(x-A)-\bar{F}(x)\}\{\bar{F}(A)-\bar{F}(x)\},
\end{aligned}
$$

hence by Lemma 3.1 and in view of the fact that $\lim _{A \rightarrow \infty} A \bar{F}(A)=0$, we have

$$
\int_{x-A}^{x}\{\bar{F}(x-y)-\bar{F}(x)\} d F(y) \sim \mu F(x, x+1] .
$$

By (3.10), (3.11) and (3.13), we obtain the desired result.

Lemma 3.4. Assume $F \in \mathscr{L}_{\Delta(t)}$ for all $t \in(0, \infty)$ and $\bar{F}^{2}(x)=o(F(x, x+1])$. Then the relation (2.3) implies $F \in \mathscr{S}_{\Delta(t)}$ for all $t \in(0, \infty)$.

Proof. Assume the relation (2.3) holds. Since $\bar{F}^{2}(x)=o(F(x, x+1])$, from Lemma 3.3 it follows that

$$
\int_{A}^{x-A}\{\bar{F}(x-y)-\bar{F}(x)\} d F(y)=o(F(x, x+1]) .
$$

Hence for $x>2 A$ and $A>t$,

$$
\begin{aligned}
\int_{A}^{x-A} F(x-y+\Delta(t)) d F(y) & \leq \int_{A}^{x-A}\{\bar{F}(x-y)-\bar{F}(x)\} d F(y) \\
& =o(F(x, x+1])=o(F(x+\Delta(t))) .
\end{aligned}
$$

Thus by lemma 3.2, we prove $F \in \mathscr{S}_{\Delta(t)}$.

Proof of Proposition 2.1 (1). We argue by induction. First the relation (2.4) is trivial for $n=2$. Furthermore, assume (2.4) holds for some $n-1 \geq 2$, i.e.,

$$
\overline{F^{(n-1) *}}(x)-(n-1) \bar{F}(x) \sim(n-1)(n-2) \mu F(x, x+1] .
$$


Then it suffices to prove (2.4) for $n$. Note that

$$
\begin{aligned}
& \overline{F^{n *}}(x)-n \bar{F}(x) \\
= & \int_{0}^{x}\left\{\overline{F^{(n-1) *}}(x-y)-(n-1) \bar{F}(x-y)\right\} d F(y) \\
& +(n-1)\left\{\overline{F^{2 *}}(x)-2 \bar{F}(x)\right\} \\
:= & I_{1}+I_{2} .
\end{aligned}
$$

Obviously,

$$
I_{2} \sim 2(n-1) \mu F(x, x+1]
$$

For $x>A>0$,

$$
\begin{aligned}
I_{1}= & \int_{0}^{x-A}\left\{\overline{F^{(n-1) *}}(x-y)-(n-1) \bar{F}(x-y)\right\} d F(y) \\
& +\int_{x-A}^{x}\left\{\overline{F^{(n-1) *}}(x-y)-(n-1) \bar{F}(x-y)\right\} d F(y) \\
:= & J_{1}+J_{2} .
\end{aligned}
$$

Since $F \in \mathscr{S}_{\Delta(1)}$, it follows from Lemma 3.2 that the relation (3.7) holds for $t=1$. Hence by (3.16), we obtain

$$
\begin{aligned}
J_{1} & \sim(n-1)(n-2) \mu \int_{0}^{x-A} F(x-y, x-y+1] d F(y) \\
& \sim(n-1)(n-2) \mu F(x, x+1] .
\end{aligned}
$$

For $J_{2}$, by integrating by parts, we obtain

$$
\begin{aligned}
J_{2}= & \int_{0}^{A}\{\bar{F}(x-y)-\bar{F}(x)\} d F^{(n-1) *}(y)-(n-1) \int_{0}^{A}\{\bar{F}(x-y)-\bar{F}(x)\} d F(y) \\
& +\left\{\overline{F^{(n-1) *}}(A)-(n-1) \bar{F}(A)\right\}\{\bar{F}(x-A)-\bar{F}(x)\} \\
:= & K_{1}-K_{2}+K_{3} .
\end{aligned}
$$

By Lemma 3.1, it follows that

$$
\begin{gathered}
K_{1} \sim \int_{0}^{\infty} y d F^{(n-1) *}(y) \cdot F(x, x+1]=(n-1) \mu F(x, x+1], \\
K_{2} \sim(n-1) \int_{0}^{\infty} y d F(y) \cdot F(x, x+1]=(n-1) \mu F(x, x+1],
\end{gathered}
$$

and

$$
K_{3} \sim\left\{\overline{F^{(n-1) *}}(A)-(n-1) \bar{F}(A)\right\} A \cdot F(x, x+1]=o(F(x, x+1]) .
$$


Then we have

$$
J_{2}=o(F(x, x+1])
$$

and hence

$$
\begin{aligned}
\overline{F^{n *}}(x)-n \bar{F}(x) \sim & (n-1)(n-2) \mu F(x, x+1] \\
+ & 2(n-1) \mu F(x, x+1]=n(n-1) \mu F(x, x+1],
\end{aligned}
$$

as required.

The proof of Proposition 2.1(2) needs the following Lemma.

Lemma 3.5. Assume $F \in \mathscr{L}_{\Delta(t)}$ for all $t \in(0, \infty), \mu<\infty$ and $\bar{F}^{2}(x)=o(F(x, x+1])$, then for all $n \geq 2$,

$$
\liminf _{x \rightarrow \infty} \frac{\overline{F^{n *}}(x)-n \bar{F}(x)}{F(x, x+1]} \geq n(n-1) \mu .
$$

Proof. We still argue by induction. In the following, we use the same notations $\left(J_{1}, I_{2}, \cdots\right)$ as in the proof of Proposition 2.1(1). From (3.11) and (3.13), it follows that for $x>2 A$,

$$
\begin{aligned}
& \int_{0}^{x}\{\bar{F}(x-y)-\bar{F}(x)\} d F(y) \\
\geq & \int_{0}^{A}\{\bar{F}(x-y)-\bar{F}(x)\} d F(y)+\int_{x-A}^{x}\{\bar{F}(x-y)-\bar{F}(x)\} d F(y) \\
\sim & 2 \mu F(x, x+1] .
\end{aligned}
$$

Then, in view of (3.10) and the condition $\bar{F}^{2}(x)=o(F(x, x+1])$, we prove (3.27) for $n=2$. Assume (3.27) holds for some $n-1 \geq 2$, i.e.,

$$
\liminf _{x \rightarrow \infty} \frac{\overline{F^{(n-1) *}}(x)-(n-1) \bar{F}(x)}{F(x, x+1]} \geq(n-1)(n-2) \mu .
$$

Then for $x>2 A$, we have

$$
\begin{aligned}
J_{1} & \gtrsim(n-1)(n-2) \mu \int_{0}^{x-A} F(x-y, x-y+1] d F(y) \\
& \geq(n-1)(n-2) \mu \int_{0}^{A} F(x-y, x-y+1] d F(y) \\
& \sim(n-1)(n-2) \mu F(x, x+1] .
\end{aligned}
$$

From the proof of Proposition 2.1(1), we have (3.25). Moreover, the relation (3.27) holds for $n=2$, i.e.

$$
I_{2}=(n-1)\left\{\overline{F^{2 *}}(x)-2 \bar{F}(x)\right\} \gtrsim 2(n-1) \mu F(x, x+1] .
$$


Hence we have

$$
\begin{aligned}
\overline{F^{n *}}(x)-n \bar{F}(x) \gtrsim \quad & (n-1)(n-2) \mu F(x, x+1] \\
+ & +2(n-1) \mu F(x, x+1]=n(n-1) \mu F(x, x+1] .
\end{aligned}
$$

Proof of Proposition 2.1(2). If the relation (2.4) holds for $n=2$, the result is obvious. Thus, we assume the relation (2.4) holds for some $n \geq 3$. From the proof of Lemma 3.5, we know that the relations from (3.29) to (3.31) still hold. However the relation (2.4) implies

$$
J_{1}+J_{2}+I_{2} \sim 2 n(n-1) \mu F(x, x+1],
$$

hence (3.30) and (3.31) necessarily hold with the sign $\gtrsim$ replaced by $\sim$. In particular, we have

$$
I_{2} \sim 2(n-1) \mu F(x, x+1]
$$

which is equivalent to (2.3). From this and Lemma 3.4, we have $F \in \mathscr{S}_{\Delta(t)}$ for all $t \in(0, \infty)$ and hence, the proof is completed.

Proof of Proposition 2.2, Without loss of generality, we assume $\varepsilon \in(0,1)$. By Lemma 3.2, we know there exist sufficiently large constants $A, A^{\prime}$ such that $A>A^{\prime}>0$ and

$$
\sup _{x \geq A}\left\{\int_{0}^{x-A^{\prime}} F(x-y, x-y+1] d F(y) \mid F(x, x+1]\right\} \leq 1+\varepsilon / 4
$$

and

$$
\sup _{x \geq A}\left\{\left|\overline{F^{2 *}}(x)-2 \bar{F}(x)\right| / F(x, x+1]\right\}<3 \mu \text {. }
$$

Obviously,

$$
\int_{0}^{x-A} F(x-y, x-y+1] d F(y) \leq \int_{0}^{x-A^{\prime}} F(x-y, x-y+1] d F(y),
$$

Hence by (3.35), we know that

$$
\sup _{x \geq A}\left\{\int_{0}^{x-A} F(x-y, x-y+1] d F(y) \mid F(x, x+1]\right\} \leq 1+\varepsilon / 4 .
$$

Since $F(\log x, \log x+1]$ is a slowly varying function, so is $1 / F(\log x, \log x+1]$, hence by Lemma 1.3.2 of Bingham et al. [6], the above $A$ can be chosen such that the function $1 / F(\log x, \log x+1]$ is locally bounded on $\left[e^{A}, \infty\right)$, i.e., $1 / F(x, x+1]$ is locally bounded on $[A, \infty)$. Hence by Lemma 3.1, we know there exists a sufficiently large constant $B>A$ such that

$$
\sup _{x \geq B}\{\{\bar{F}(x-A)-\bar{F}(x)\} / F(x, x+1]\}<\infty
$$


and

$$
\sup _{A \leq x<B}\{\{\bar{F}(x-A)-\bar{F}(x)\} / F(x, x+1]\} \leq \sup _{A \leq x<B}\{1 / F(x, x+1]\}<\infty .
$$

Thus, there exists a positive constant $M$, which is independent of $n$, such that both the left-hand sides of (3.39) and (3.40) do not exceed $M$. On the other hand, by the definition of $J_{2}$, it is easy to see

$$
\left|J_{2}\right| \leq n\{\bar{F}(x-A)-\bar{F}(x)\}
$$

Hence we have

$$
\begin{aligned}
\sup _{x \geq A}\left\{\left|J_{2}\right| / F(x, x+1]\right\} & \leq n \sup _{x \geq A}\{\{\bar{F}(x-A)-\bar{F}(x)\} / F(x, x+1]\} \\
& \leq M n<\infty .
\end{aligned}
$$

Denote

$$
\alpha_{n}=\sup _{x \geq A}\left|\frac{\overline{F^{n *}}(x)-n \bar{F}(x)}{F(x, x+1]}\right| .
$$

By (3.38), we have

$$
\begin{aligned}
& \sup _{x \geq A}\left\{\left|J_{1}\right| / F(x, x+1]\right\} \\
\leq & \alpha_{n-1} \sup _{x \geq A}\left\{\int_{0}^{x-A} F(x-y, x-y+1] d F(y) \mid F(x, x+1]\right\} \\
\leq & (1+\varepsilon / 4) \alpha_{n-1} .
\end{aligned}
$$

From (3.36), (3.42) and (3.44) it follows that

$$
\alpha_{n} \leq(1+\varepsilon / 4) \alpha_{n-1}+3 \mu(n-1)+M n \leq(1+\varepsilon / 4) \alpha_{n-1}+C_{1} n,
$$

where $C_{1}=3 \mu+M$. By induction and in view of $\alpha_{1}=0$, we obtain

$$
\alpha_{n} \leq C_{1} \sum_{i=0}^{n-2}(n-i)(1+\varepsilon / 4)^{i} \leq C_{1} n^{2}(1+\varepsilon / 4)^{n},
$$

It is easy to see that the right-hand side of (3.46) does not exceed $K(1+\varepsilon)^{n}$ for an appropriately chosen constant $K$ and hence, the proof is completed.

Let

$$
\beta_{n}=\inf _{x \geq A} \frac{\overline{F^{n *}}(x)-n \bar{F}(x)}{F(x, x+1]} .
$$


Lemma 3.6. Assume $F$ is a distribution on $[0, \infty)$ satisfying $\bar{F}^{2}(x)=o(F(x, x+1])$. Then there exists a constant $A>0$, which is independent of $n$, such that for all $n \geq 2$,

$$
\beta_{n} \geq-n^{2}
$$

Proof. By Bonfferoni's inequality, we have

$$
\begin{aligned}
\overline{F^{n *}}(x) & =P\left(S_{n}>x\right) \\
& \geq P\left(\max _{1 \leq k \leq n} X_{k}>x\right) \\
& \geq \sum_{k=1}^{n} P\left(X_{k}>x\right)-\sum_{1 \leq i<j \leq n} P\left(X_{i}>x, X_{j}>x\right) \\
& \geq n \bar{F}(x)-n^{2} \bar{F}^{2}(x),
\end{aligned}
$$

Since $\bar{F}^{2}(x)=o(F(x, x+1])$, there exists a sufficiently large constant $A>0$ such that

$$
\sup _{x \geq A}\left\{\bar{F}^{2}(x) / F(x, x+1]\right\} \leq 1 \text {. }
$$

Combining (3.49) and (3.50) gives (3.48).

Proof of Theorem 2.1, (1) By Proposition 2.1 (1), Proposition 2.2 and the dominated convergence theorem, we obtain the desired result.

(2) Obviously,

$$
\begin{aligned}
& p_{l} \limsup _{x \rightarrow \infty} \frac{\overline{F^{l *}}(x)-l \bar{F}(x)}{F(x, x+1]} \\
\leq & \lim _{x \rightarrow \infty} \frac{\bar{G}(x)-\left(\sum_{n=0}^{\infty} n p_{n}\right) \bar{F}(x)}{F(x, x+1]}-\liminf _{x \rightarrow \infty} \frac{\sum_{n \neq l}\left\{\overline{F^{n *}}(x)-n \bar{F}(x)\right\} p_{n}}{F(x, x+1]} \\
= & \mu \sum_{n=0}^{\infty} n(n-1) p_{n}-\liminf _{x \rightarrow \infty} \frac{\sum_{n \neq l}\left\{\overline{F^{n *}}(x)-n \bar{F}(x)\right\} p_{n}}{F(x, x+1]} .
\end{aligned}
$$

By Lemma 3.6, we know that Fatou's Lemma (cf. p. 94 of Chow and Teicher [8] ) can be applied to the second term above, which gives

$$
\begin{aligned}
& \liminf _{x \rightarrow \infty} \frac{\sum_{n \neq l}\left\{\overline{F^{n *}}(x)-n \bar{F}(x)\right\} p_{n}}{F(x, x+1]} \\
\geq & \sum_{n \neq l} \liminf _{x \rightarrow \infty}\left\{\frac{\overline{F^{n *}}(x)-n \bar{F}(x)}{F(x, x+1]}\right\} p_{n} \\
\geq & \mu \sum_{n \neq l} n(n-1) p_{n},
\end{aligned}
$$


where in the last step, Lemma 3.5 has been applied. Combining (3.51) and (3.52) gives

$$
\begin{aligned}
p_{l} \limsup _{x \rightarrow \infty} \frac{\overline{F^{l *}}(x)-l \bar{F}(x)}{F(x, x+1]} & \leq \mu \sum_{n=0}^{\infty} n(n-1) p_{n}-\mu \sum_{n \neq l} n(n-1) p_{n} \\
& =p_{l} l(l-1) \mu .
\end{aligned}
$$

From this and Lemma 3.5 it follows that

$$
\lim _{x \rightarrow \infty} \frac{\overline{F^{l *}}(x)-l \bar{F}(x)}{F(x, x+1]}=l(l-1) \mu .
$$

Hence by Proposition 2.1(2), we obtain $F \in \mathscr{S}_{2}$.

Proof of Proposition 2.3, Notice that

$$
\frac{H(x+\Delta(t))}{K \cdot F(x+\Delta(t))}-1=\frac{\bar{H}(x)-K \cdot \bar{F}(x)}{K \cdot F(x+\Delta(t))}-\frac{\bar{H}(x+t)-K \cdot \bar{F}(x+t)}{K \cdot F(x+\Delta(t))}
$$

and

$$
F(x+t+\Delta(t)) \sim F(x+\Delta(t)) .
$$

Hence by (2.7) and Lemma 3.1, we know that the right-hand side of (3.55) tends to zero, i.e.,

$$
H(x+\Delta(t)) \sim K \cdot F(x+\Delta(t)) .
$$

Hence by Lemma 1 of Asmussen et al. [1], we have

$$
H \in \mathscr{S}_{\Delta(t)} .
$$

By (2.7),

$$
\bar{H}^{2}(x)-K^{2} \cdot \bar{F}^{2}(x)=\{\bar{H}(x)-K \cdot \bar{F}(x)\}\{\bar{H}(x)+K \cdot \bar{F}(x)\}=o(F(x, x+1]) .
$$

Notice that

$$
\begin{aligned}
& \int_{A}^{x-A}\{\bar{H}(x-y)-\bar{H}(x)\} d F(y) \\
= & K \int_{A}^{x-A}\{\bar{F}(x-y)-\bar{F}(x)\} d F(y)+\int_{A}^{x-A}\{\bar{H}(x-y)-K \cdot \bar{F}(x-y)\} d F(y) \\
& -\int_{A}^{x-A}\{\bar{H}(x)-K \cdot \bar{F}(x)\} d F(y) .
\end{aligned}
$$

By (2.7), we have

$$
\int_{A}^{x-A}\{\bar{H}(x)-K \cdot \bar{F}(x)\} d F(y) \leq\{\bar{H}(x)-K \cdot \bar{F}(x)\} \bar{F}(A)=o(F(x, x+1])
$$


and

$$
\begin{aligned}
& \int_{A}^{x-A}\{\bar{H}(x-y)-K \cdot \bar{F}(x-y)\} d F(y) \\
\sim & c \int_{A}^{x-A} \bar{F}(x-y, x-y+1] d F(y)=o(F(x, x+1]),
\end{aligned}
$$

where in the second step, Lemma 3.2 is applied since $F \in \mathscr{S}_{\Delta(t)}$. Substituting (3.61) and (3.62) into (3.60), we obtain

$$
\begin{aligned}
& \int_{A}^{x-A}\{\bar{H}(x-y)-\bar{H}(x)\} d F(y)=K \int_{A}^{x-A}\{\bar{F}(x-y)-\bar{F}(x)\} d F(y) \\
& +o(F(x, x+1]) \text {. }
\end{aligned}
$$

For the same reason, in view of (3.57) and (3.58), we obtain

$$
\int_{A}^{x-A}\{\bar{H}(x-y)-\bar{H}(x)\} d H(y)=\underset{+}{K \int_{A}^{x-A}\{\bar{F}(x-y)-\bar{F}(x)\} d H(y)}
$$

By integrating by parts, we have

$$
\begin{aligned}
& \int_{A}^{x-A}\{\bar{F}(x-y)-\bar{F}(x)\} d H(y) \\
= & \int_{A}^{x-A}\{\bar{H}(x-y)-\bar{H}(x)\} d F(y)+\{\bar{F}(x-A)-\bar{F}(x)\}\{\bar{H}(A)-\bar{H}(x-A)\} \\
& -\{\bar{H}(x-A)-\bar{H}(x)\}\{\bar{F}(A)-\bar{F}(x-A)\},
\end{aligned}
$$

hence by (3.57) and Lemma 3.1, we obtain

$$
\begin{aligned}
& \int_{A}^{x-A}\{\bar{F}(x-y)-\bar{F}(x)\} d H(y) \\
= & \int_{A}^{x-A}\{\bar{H}(x-y)-\bar{H}(x)\} d F(y)+o(F(x, x+1]) .
\end{aligned}
$$

Then from (3.63), (3.64), (3.66) and (3.59) it follows that

$$
\begin{aligned}
& \int_{A}^{x-A}\{\bar{H}(x-y)-\bar{H}(x)\} d H(y)-\bar{H}^{2}(x) \\
= & K^{2}\left\{\int_{A}^{x-A}\{\bar{F}(x-y)-\bar{F}(x)\} d F(y)-\bar{F}^{2}(x)\right\}+o(F(x, x+1]) .
\end{aligned}
$$

Thus by Lemma 3.3 and (3.57), we conclude that $H \in \mathscr{S}_{2}$.

Proof of Lemma 2.1. Firstly, it is easy to see

$$
\int_{0}^{x} F(s, s+t] d s=\int_{0}^{t} \bar{F}(s) d s-\int_{x}^{x+t} \bar{F}(s) d s .
$$


Let $x \rightarrow \infty$ in (3.68), we obtain

$$
\int_{0}^{\infty} F(s, s+t] d t=\int_{0}^{t} \bar{F}(s) d s<\infty,
$$

and thus $K F(\cdot+\Delta(t))$ is a density function. From Proposition 2, the proof of Lemma 1 of Asmussen et al. [1], it is easy to see that $F \in \mathscr{S}_{\Delta(t)}$ is equivalent to that $F \in \mathscr{L}_{\Delta(t)}$ and for every function $l(x)$ such that $l(x) \rightarrow \infty$ and $l(x)<x / 2$, the following relation holds:

$$
\int_{l(x)}^{x-l(x)} F(x-y+\Delta(t)) d F(y)=o(F(x+\Delta(t))), \quad x \rightarrow \infty .
$$

Note that if (3.70) holds with $l(x)$ replaced by some $l_{1}(x)$ such that $l_{1}(x)<l(x)$, then (3.70) itself holds. Hence without loss of generality, we assume $t$ divides exactly $x-2 l(x)$ and denote $n(x)=(x-2 l(x)) / t$. Assume $F \in \mathscr{L}_{\Delta(t)}$. Then we have

$$
\begin{aligned}
& \int_{l(x)}^{x-l(x)} F(x-y+\Delta(t)) d F(y) \\
= & \sum_{k=1}^{n} \int_{l(x)+(k-1) t}^{l(x)+k t} F(x-y+\Delta(t)) d F(y) \\
\sim & \sum_{k=1}^{n} F(x-l(x)-(k-1) t+\Delta(t)) F(l(x)+(k-1) t+\Delta(t)) \\
\sim & \frac{1}{t} \sum_{k=1}^{n} \int_{l(x)+(k-1) t}^{l(x)+k t} F(x-y+\Delta(t)) F(y+\Delta(t)) d y \\
= & \frac{1}{t} \int_{l(x)}^{x-l(x)} F(x-y+\Delta(t)) F(y+\Delta(t)) d y, \quad x \rightarrow \infty .
\end{aligned}
$$

Thus, by Proposition 6 of Asmussen et al. [1], we prove the desired result.

Proof of Proposition 2.4, Note that

$$
\begin{aligned}
& \int_{-1}^{y} h(x-t-1) d t \\
= & \int_{y}^{y+1} \bar{F}(x-t) d t-\int_{-1}^{0} \bar{F}(x-t) d t \\
\geq & \bar{F}(x-y)-\bar{F}(x),
\end{aligned}
$$

hence,

$$
\begin{aligned}
& \int_{A}^{x / 2}\{\bar{F}(x-y)-\bar{F}(x)\} d F(y) \\
\leq & \int_{A}^{x / 2} \int_{-1}^{y} h(x-t-1) d t d F(y) \\
\leq & \bar{F}(A) \int_{-1}^{A} h(x-t-1) d t+\int_{A}^{x / 2} h(x-t-1) \bar{F}(t) d t \\
:= & V_{1}+V_{2},
\end{aligned}
$$


where in the second step, Fubini's theorem is applied to interchange the order of integration. It is easy to see

$$
V_{1} \sim A \bar{F}(A) h(x)=o(h(x))
$$

Since

$$
\int_{0}^{x} \bar{F}(x-t) \bar{F}(t) d t=2 \int_{0}^{x / 2} \bar{F}(x-t) \bar{F}(t) d t,
$$

it is easy to see that $F \in \mathscr{S}^{*}$ implies

$$
\int_{A}^{x / 2} \bar{F}(x-t) \bar{F}(t) d t=o(\bar{F}(x)) .
$$

By Theorem 2.0.8 in Bingham et al. [6], (2.13) holds locally uniformly in $(0, \infty)$. Hence

$$
\begin{aligned}
V_{2} & \sim \int_{A}^{x / 2} h(x-t) \bar{F}(t) d t \\
& =\int_{A}^{x / 2} q(x-t) \bar{F}(x-t) \bar{F}(t) d t \\
& \lesssim q(x) \int_{A}^{x / 2} \bar{F}(x-t) \bar{F}(t) d t=o(h(x)) .
\end{aligned}
$$

Combining (3.74) and (3.77) gives

$$
\int_{A}^{x / 2}\{\bar{F}(x-y)-\bar{F}(x)\} d F(y)=o(h(x)) .
$$

Hence by integrating by parts and using $\bar{F}^{2}(x / 2)=o(h(x))$, we have

$$
\int_{x / 2}^{x-A}\{\bar{F}(x-y)-\bar{F}(x)\} d F(y)=\int_{A}^{x / 2}\{\bar{F}(x-y)-\bar{F}(x)\} d F(y)+o(h(x)) .
$$

From this and (3.78), it follows that

$$
\int_{A}^{x-A}\{\bar{F}(x-y)-\bar{F}(x)\} d F(y)=o(h(x)) .
$$

Note that $\bar{F}^{2}(x) \leq \bar{F}^{2}(x / 2)=o(h(x))$, hence by Lemma 3.3, we prove (2.3). From this and Lemma 3.4, it follows that $F \in \mathscr{S}_{\Delta(t)}$ for all $t>0$ and hence, the proof is completed.

Proof of Corollary 2.1, By (3.72) and (2.14), we have

$$
\begin{aligned}
& \int_{A}^{x / 2}\{\bar{F}(x-y)-\bar{F}(x)\} d F(y) \\
\leq & \int_{A}^{x / 2} \int_{-1}^{y} h(x-t-1) d t d F(y) \\
\lesssim & h(x) \int_{A}^{x / 2} y d F(y)=o(h(x)),
\end{aligned}
$$


i.e. the relation (3.78) holds. The remaining proof is similar to that of Proposition 2.4 and we omit it.

\section{Acknowledgements}

This research is supported by NNSF (grant No. 10926043) in China. The author is also thankful to the anonymous referee for his/her helpful comments which results in the improvement of this paper.

\section{References}

[1] Asmussen, S, Foss, S. And Korshunov, D. (2003). Asymptotics for sums of random variables with local subexponential behaviour. Journal of Theoretical Probability, 16, 489518.

[2] Baltrūnas, A. (1999). Second order behaviour of ruin probabilities. Scand. Actuarial J. 2, $120-133$.

[3] Baltrūnas, A., Omey, E. (1998). The rate of convergence for subexponential distributions. Liet. Matem. Rink. 38(1), 1-18.

[4] Baltrūnas, A., OMey, E. (2002). The rate of convergence for subexponential distributions and densities. Lithuanian Mathematical Journal 42, 1-14.

[5] Baltrūnas, A., Omey, E., Van Gulck, S. (2006). Hazard rates and subexponential distributions. Publications de L' Institut Mathématique, nouvelle série, tome 80, 29-46.

[6] Bingham, N.B., Goldie, C.M. and Teugels, J.L. (1987). Regular Variation. Cambridge: Cambridge University Press.

[7] Chistyakov, V.P. (1964). A theorem on sums of independent positive random variables and its application to branching random processes. Theory Probab. Appl. 9, 640-648.

[8] Chow, Y.S. and Teicher, H. (1978). Probability Theory. Springer. New York.

[9] Geluk, J. L. and Pakes, A. G. (1991). Second order subexponential distributions.J. Austral. Math. Soc. (Series A)., 51, 73-87.

[10] Geluk, J. L. (1992). Second order tail behaviour of a subordinated probability distribution. Stochastic Processes and their Applications 40, 325-337 
[11] Geluk, J. L. (1996). Tails of subordinated laws: The regularly varying case. Stochastic Processes and their Applications 61, 147-161.

[12] KlüPPELBERG,C. 1988. Subexponential distributions and integrated tails. Journal of applied probability, 25, 132-141.

[13] Omey, E. AND Willekens, E. (1986). Second order behaviour of the tail of a subordinated probability distribution. stochastic processes and their applications $\mathbf{2 1 , 3 3 9 - 3 5 1 . ~}$

[14] Omey, E. And Willekens, E. (1987). Second-order behaviour of distributions subordinate to distribution with finite mean. Commun. Statist. Stochastic Models. 3, 311-342.

[15] OMEY, E. (1994). On the difference between the product and the convolution product of distribution functions. Publications de L'Institut Mathématique, nouvelle série, tome, 55(69), $111-145$. 